\title{
Impacts d'une crue majeure sur l'alimentation en eau potable de Paris et de l'Ile de France
}

\author{
Impacts of a major flood on the drinking \\ water distribution in Paris and Ile-de-France \\ par J. Cavard \\ Syndicat des Eaux d'Ile de France \\ D. Giraud \\ Sagep - Eau de Paris \\ J-L. Hamon \\ Compagnie Générale des Eaux \\ J-P. Maugendre \\ Lyonnaise des Eaux
}

The drinking water distributors in lle de France regularly have to face river floods. In the last decades heavy floods were observed (in 1955, 1982, 1988...) but thanks to protective measures implemented during this period, no inconvenience to the consumers was recorded.

Nevertheless, some facilities would be definitely flooded in case of a major flood like that of 1910. With, as a result, a noticeable reduction of the potential of water production capacity.

Subsequently, in addition to the direct impact of the submersion of the facilities, the water distributors would also have to take into account the resulting adverse effect on the economical and physical environment of the water treatment plants ; this would increase their vulnerability (raw water quality, power supply, reagents supply...).

These different aspects are discussed here.

I $\square$ VULNÉRABILITÉ DES USINES DE PRODUCTION D'EAU POTABLE VIS-A-VIS DE LA MONTÉE DES EAUX (D. Giraud - SAGEP)

\subsection{La distribution de l'eau potable en Ile de France}

Chaque jour, la production d'eau potable nécessaire à la satisfaction des besoins des usagers d'Ile de France est de $2100000 \mathrm{~m}^{3}$.

Pour réaliser cette production, le SEDIF, la Lyonnaise des Eaux à l'extérieur de Paris et la SAGEP pour la Capitale, utilisent un grand nombre de sites, comme le montre le tableau 1:

La région Ile de France dispose par conséquent d'un potentiel de plus de 4 millions de $\mathrm{m}^{3} / \mathrm{j}$ pour alimenter les réseaux interconnectés de l'ensemble de ce secteur.

$19 \%$ eau souterraine,

$7 \%$ eau souterraine avec réalimentation artificielle par eau de rivière,

$74 \%$ eau de surface (62\% par la Seine, $31 \%$ par la Marne et $7 \%$ par l'Oise),
Cette grande diversité constitue sans aucun doute le point fort de la sécurité pour l'approvisionnement en eau potable de l'Ile de France dans la mesure où tous les réseaux sont interconnectés. Toutefois, dans le cadre d'une crue exceptionnelle, il est vraisemblable que l'ensemble des rivières de la région serait affecté et toutes les usines d'eau potable seraient susceptibles de connaître des difficultés.

\subsection{Protection des différents sites de production en cas de crue}

Les niveaux des rivières atteints en 1910 puis en 1924 et 1955 ont conduit les concepteurs des usines de traitement d'eau de surface à les protéger efficacement contre la montée des eaux. Beaucoup d'entre elles ont été construites afin de les mettre à l'abri d'une crue de type 1910 ; pour d'autres, les cotes ont été calées sur le niveau de la crue de 1924.

Toutefois, il s'agit d'une protection vis-à-vis de l'inondation directe des installations. Il faut souvent aussi protéger les équipements contre les infiltrations dans le site par la remontée des nappes. Mais il est difficile de connaître l'efficacité réelle des dispositifs de pompage mis en place à cet 


\begin{tabular}{|c|c|c|c|c|c|c|}
\hline & & Seine & Marne & Oise & $\begin{array}{l}\text { Eau souterraine } \\
\text { avec réalimentation } \\
\text { par eau de rivière }\end{array}$ & Eau souterraine \\
\hline SEDIF & $\begin{array}{l}\text { Choisy le Roi } \\
\text { Neuilly sur Marne } \\
\text { Noisy le Grand } \\
\text { Mery sur Oise } \\
\text { Aulnay } \\
\text { Pantin } \\
\text { Neuilly sur Seine } \\
\text { Adduct. Champigny }\end{array}$ & 650000 & 600000 & 200000 & & $\begin{array}{c}12000 \\
4300 \\
8100 \\
80000\end{array}$ \\
\hline L.E. & $\begin{array}{l}\text { Morsang sur Seine } \\
\text { Viry Chatillon } \\
\text { Vigneux sur Seine } \\
\text { Champigny } \\
\text { Corbeil Essonnes } \\
\text { Mont-Valérien } \\
\text { Villeneuve la Garenne } \\
\text { Le Pecq - Croissy } \\
\text { Poissy Migneaux } \\
\text { Vernouillet } \\
\text { Flins Aubergenville } \\
\text { La Chapelle } \\
\text { Captages divers }\end{array}$ & $\begin{array}{c}225000 \\
120000 \\
55000 \\
24000 \\
115000 \\
\\
3000 \\
7000\end{array}$ & & & $\begin{array}{l}158000 \\
132000\end{array}$ & $\begin{array}{l}40000 \\
40000 \\
15000 \\
25000\end{array}$ \\
\hline SAGEP & $\begin{array}{l}\text { Ivry } \\
\text { Orly } \\
\text { Joinville } \\
\text { Dérivations Sud } \\
\text { Dérivations Ouest }\end{array}$ & $\begin{array}{l}300000 \\
300000\end{array}$ & 300000 & & & $\begin{array}{l}360000 \\
160000\end{array}$ \\
\hline Total & & 1799000 & 900000 & 200000 & 290000 & 744400 \\
\hline
\end{tabular}

Tableau 1. - Principaux sites de production en lle de France.

effet. Or les conditions de fonctionnement des usines de traitement d'eau dépendent essentiellement de la mise hors d'eau des points sensibles.

En dehors de ces dispositifs installés à demeure, certaines mesures sont à prendre lors de la montée des eaux. A cette fin, les sites disposent généralement de cahiers de consignes de crues qui détaillent les dispositions à mettre en œuvre au fur et à mesure de la progression de la montée des eaux. Il s'agit principalement de :

- la mise en place de pompages d'épuisement complémentaires pour évacuer les infiltrations,

- l'obturation des passages permettant des venues d'eau (chemin de câbles, ....),

- la construction de murets de protection devant certains accès.

Enfin, on signalera que de nombreuses usines disposent de groupes électrogènes fonctionnant au gas-oil permettant d'assurer la production d'eau potable en cas de défaillance du réseau EDF. C'est le cas de trois usines de la SAGEP (Ivry, Orly et Joinville) mais dans ces conditions de fonctionnement, la capacité de l'usine est limitée à $200000 \mathrm{~m} 3 / \mathrm{j}$ (par rapport à $300000 \mathrm{~m}^{3} / \mathrm{j}$ lorsque l'alimentation se fait par le réseau EDF).

\subsection{Bilans de production en cas de crue}

Pour réaliser ces bilans, on prend en compte deux niveaux de crues :

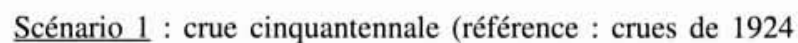
et 1955)

Scénario 2 : crue centennale (référence : crue de 1910).

On tiendra compte par ailleurs des aménagements réalisés depuis l'époque de ces crues en prenant comme hypothèse un abaissement des cotes compris entre 0,5 et $1 \mathrm{~m}$.

L'observation de ces bilans montre une situation relativement confortable, en particulier dans le scénario 1 , où le potentiel de production n'est réduit que de $9 \%$. Pour le scénario 2 , qui correspond à la crue historique la plus importante connue, la capacité de production des sites pour la région Ile de France est abaissée de $32 \%$. On notera que le potentiel de production est de $2,6 \mathrm{Mm}^{3}$ pour une consommation de $2,1 \mathrm{Mm}^{3}$, ce qui laisse encore un coefficient de sécurité de 1,2 .

On peut cependant penser que dans le cadre d'un événement d'une telle ampleur, la demande en eau serait fortement réduite. En effet, la région Ile de France se trouverait concernée bien au-delà de la production de l'eau potable et il est vraisemblable que toute la zone inondée serait paralysée. La réduction de l'activité économique due, entre autres, aux problèmes de transport, entraînerait une baisse importante de la consommation d'eau potable. Le chiffre de $25 \%$ de réduction de consommation d'eau potable du réseau paraît réaliste (c'est cette valeur qui avait été prise comme hypothèse dans le scénario de l'exercice de crise régional LUTECE 95). 


\begin{tabular}{|c|c|c|c|c|}
\hline & & Capacité nominale & $\begin{array}{c}\text { Scénario } 1 \\
1924-1955\end{array}$ & $\begin{array}{c}\text { Scénario } 2 \\
1910\end{array}$ \\
\hline SEDIF & $\begin{array}{l}\text { Choisy le Roi } \\
\text { Neuilly sur Marne / } \\
\text { Noisy le Grand } \\
\text { Mery sur Oise } \\
\text { Aulnay } \\
\text { Pantin } \\
\text { Neuilly sur Seine } \\
\text { Adduct. Champigny }\end{array}$ & $\begin{array}{r}650000 \\
600000 \\
200000 \\
12000 \\
4300 \\
8100 \\
80000\end{array}$ & $\begin{array}{r}650000 \\
600000 \\
\\
200000 \\
12000 \\
4300 \\
8100 \\
80000\end{array}$ & $\begin{array}{r}650000 \\
600000 \\
\\
200000 \\
12000 \\
4300 \\
8100 \\
80000\end{array}$ \\
\hline L.E. & $\begin{array}{l}\text { Morsang sur Seine } \\
\text { Viry Chatillon } \\
\text { Vigneux sur Seine } \\
\text { Champigny } \\
\text { Corbeil Essonnes } \\
\text { Mont-ValËrien } \\
\text { Villeneuve la Garenne } \\
\text { Le Pecq ve la Gare } \\
\text { Poissy Migneaux } \\
\text { Vernouillet } \\
\text { Flins Aubergenville } \\
\text { La Chapelle } \\
\text { Captages divers }\end{array}$ & $\begin{array}{r}225000 \\
120000 \\
55000 \\
40000 \\
24000 \\
115000 \\
40000 \\
158000 \\
3000 \\
7000 \\
132000 \\
15000 \\
25000\end{array}$ & $\begin{array}{r}225000 \\
0 \\
0 \\
40000 \\
24000 \\
70000 \\
15000 \\
120000 \\
0 \\
0 \\
61000 \\
15000 \\
25000\end{array}$ & $\begin{array}{r}150000 \\
0 \\
0 \\
40000 \\
15000 \\
70000 \\
5000 \\
37000 \\
0 \\
0 \\
53000 \\
15000 \\
25000\end{array}$ \\
\hline SAGEP & $\begin{array}{l}\text { Ivry } \\
\text { Orly } \\
\text { Joinville } \\
\text { Dérivations Sud } \\
\text { Dérivations Ouest }\end{array}$ & $\begin{array}{l}300000 \\
300000 \\
300000 \\
360000 \\
160000\end{array}$ & $\begin{array}{l}300000 \\
300000 \\
300000 \\
360000 \\
160000\end{array}$ & $\begin{array}{r}300000 \\
0 \\
280000 \\
100000\end{array}$ \\
\hline Total & & 3933400 & 3569400 & 2644400 \\
\hline
\end{tabular}

Tableau 2. - Réduction des capacités de production en cas de crue.

Le tableau 3 montre que dans ces conditions, chaque grand secteur de distribution disposerait de la capacité de production nécessaire à la satisfaction des besoins des usagers. Par ailleurs, les interconnexions pourraient être mises en œuvre pour secourir les parties de réseau les moins bien approvisionnées.

Enfin il faut noter que, dans l'hypothèse du scénario 2 (crue de type 1910), la phase la plus pénalisante ne durerait que quelques jours et les capacités de stockage des réservoirs $\left(2,1 \mathrm{Mm}^{3}\right)$ pourraient servir de tampon pour effacer les périodes les plus critiques.

II — L'ALIMENTATION EN EAU POTABLE : DÉPENDANTE DE LA QUALITÉ DES RESSOURCES, ET DU FONCTIONNEMENT DES AUTRES RÉSEAUX RÉGIONAUX

(J.L. Hamon - CGE et J. Cavard - SEDIF)
La bonne protection face aux inondations d'une station de production d'eau potable ne doit cependant pas laisser supposer que celle-ci puisse fonctionner sans désordre en cas de crue majeure : la production d'eau potable est un processus industriel dépendant de la disponibilité et de la qualité des ses matières premières (eau brute, énergie, réactifs, ...) et sa distribution s'appuie sur un réseau physique lui aussi vulnérable. L'alimentation en eau serait donc particulièrement sensible aux facteurs de dysfonctionnement physiques et économiques qui affecteraient l'Ile de France en cas de crue majeure, dont nous présentons ici une liste sans doute non exhaustive.

2. 1 Vulnérabilité du processus de production d'eau potable

a) La qualité de la ressource

La première préoccupation des producteurs d'eau potable en période de crue est la dégradation de la ressource. Sur la

\begin{tabular}{|l|r|r|r|r|c|}
\hline & Capacité nominale & $\begin{array}{c}\text { Scénario 1 } \\
1924-1955\end{array}$ & $\begin{array}{c}\text { Scénario 2 } \\
1910\end{array}$ & $\begin{array}{c}\text { Consommation } \\
\text { moyenne }\end{array}$ & $\begin{array}{c}\text { Consommation } \\
\text { pendant la } \\
\text { Crue de 1910 }\end{array}$ \\
\hline SEDIF & 1554400 & 1554400 & 1554400 & 900000 & 675000 \\
\hline L.E. & 959000 & 595000 & 410000 & 500000 & 375000 \\
\hline SAGEP & 1420000 & 1420000 & 680000 & 700000 & 525000 \\
\hline Total & $\mathbf{3 9 3 3 4 0 0}$ & $\mathbf{3 5 6 9 4 0 0}$ & $\mathbf{2 6 4 4} \mathbf{4 0 0}$ & $\mathbf{2 1 0 0 0 0 0}$ & $\mathbf{1 5 7 5 0 0 0}$ \\
\hline
\end{tabular}

Tableau 3. - Bilans besoins-ressources. 
Seine, la Marne et l'Oise, cette dégradation est importante, même pour les crues modestes observées lors de la dernière décennie (fig 1 et 2 ), et impacte naturellement en tout premier lieu les usines procédant à des prélèvements directs en rivière.

L'impact qualitatif des crues se traduit tout d'abord par de fortes concentrations en matières en suspension et une forte turbidité qui nécessitent un renforcement des taux de coagulant. Ces teneurs importantes en matières en suspension sont dues aux écoulements superficiels consécutifs aux précipitations sur les bassins versants ainsi qu'à la remise en suspension des sédiments dans les lits des rivières, voire à l'érosion provoquée par les cours d'eau eux-mêmes.

Cette augmentation des matières en suspension s'accompagne d'une augmentation de la teneur en matières organiques, caractérisée par le carbone organique total (C.O.T.), de l'ammonium, des nitrates et des pesticides.

A ces pollutions d'origine diffuse peuvent se conjuguer une série de pollutions ponctuelles accidentelles causées par des délestages de stations d'épuration ou par l'inondation d'entrepôts et de sites industriels, présents en grand nombre à proximité des rivières d'Ile de France. Heureusement, l'impact de ces pollutions accidentelles se trouve limité par l'effet de dilution important lié au débit des rivières.

$\mathrm{Si}$ les ordres de grandeur des variations qualitatives de la ressource en période de fort débit et les sites potentiellement générateurs de pollutions accidentelles sont bien connus des distributeurs, il est peu aisé d'extrapoler ces données dans des scénarios de concomitance exceptionnelle de tous les facteurs de pollution possibles. Il est cependant sûr que les capacités nominales de traitement des stations d'eau potable seraient fortement réduites par l'exigence de traitements plus poussés et que la distribution de l'eau produite, tout en ne présentant vraisemblablement aucun risque pour la santé publique, appellerait à des dérogations sanitaires.

Il est de plus à noter que les exceptionnels débits des rivières, sorties de leurs lits mineurs, rendraient quasiment inopérants les outils de prévision de la propagation des pollutions (DISPERSO, POLLUX) qui n'ont par nature été calés que pour des plages habituelles de conditions hydrométriques.

\section{b) L'alimentation en énergie électrique}

L'étude menée par les Grands Lacs de Seine en 1994 a montré qu'une importante crue de la Seine affecterait de nombreux postes de transformation électriques, voire une centrale EDF. Même si toutes les grandes stations d'eau potable de l'Ile de France disposent d'au moins deux alimentations électriques distinctes provenant de postes sources différents, il n'est pas certain que les conditions générales de fourniture d'énergie soient suffisantes pour les faire fonctionner.

De plus, il est loin d'être assuré que les plans de coupure qui seraient mis en cuvre privilégieraient l'alimentation en eau potable face à d'autres activités régionales, économiques ou stratégiques.

Seules quelques grandes installations d'Ile de France disposent d'une autonomie de production d'électricité par groupes électrogènes : la station du SEPG au Mont Valérien et les trois usines de la SAGEP, qui verraient alors leur production limitée aux deux tiers de leur capacité (200 000 $\mathrm{m}^{3} / \mathrm{j}$ ). Ces groupes électrogènes ayant principalement été installés pour des raisons d'optimisation du coût de l'énergie (contrats dits EJP) ne sont cependant pas dotés de réserves de carburant leur permettant de fonctionner au-delà de quelques jours. Il s'agirait alors de s'assurer que le réseau routier permet leur accessibilité et que carburants et véhicules-citernes sont disponibles.

\section{c) L'approvisionnement en réactifs chimiques}

Les stations de production d'eau potable disposent sur leurs sites de stocks importants de réactifs chimiques, permettant une autonomie de plusieurs semaines en situation normale. L'utilisation à forte dose de certains d'entre eux en cas de crue, notamment des coagulants, limiterait cependant la durée de cette autonomie et le fonctionnement des stations se trouverait alors gravement compromis en cas d'impossibilité de réapprovisionnement. Des situations critiques furent ainsi frôlées lors de récentes grèves des transporteurs routiers. La montée progressive des eaux peut laisser au producteur le temps d'augmenter préventivement ses stocks, mais là encore, il s'agit que la station demeure accessible par voie routière.

\section{d) L'accessibilité aux installations}

En effet, le fait que le gros cuvre d'une station de production d'eau potable soit hors d'eau n'exclut pas pour autant que l'inondabilité des voies de communication ou d'accès aux installations à l'intérieur du site. A titre d'exemple, les quais bordant l'usine du SEDIF à Choisy-le-Roi, elle-même protégée face à une crue de type 1910 , sont inondables à partir de la cote $36,20 \mathrm{~m}$ (crue décennale).

Les camions de livraison doivent alors passer par un pont SNCF limité à 16 tonnes ce qui pourrait obliger, sauf dérogation, à des transbordements de réactifs dans des citernes plus petites. Alors même que la station Lyonnaise des Eaux de Morsang-sur-Seine pourrait fonctionner en cas de crue de type 1910, grâce à une adduction d'eau de l'Essonne compensant la mise hors service de la prise d'eau en Seine, les voies intérieures à son site se trouveraient vraisemblablement inondées sur quelques mètres. D'une manière générale, il est à prévoir d'importantes difficultés d'accessibilité et de déplacement pour les agents responsables du service de la production.

e) L'alimentation en courants faibles

La conduite des installations de distribution d'eau repose en grande partie sur des automatismes locaux, des télécommandes, télécontrôles et télémesures. Des liaisons courants faibles en triangle permettent de s'affranchir de l'indisponibilité temporaire et localisée d'une liaison. En revanche, une défaillance plus généralisée des réseaux de communication rendrait la conduite des installations très délicate et peu optimale et nécessiterait une mobilisation importante du personnel.

La défaillance des réseaux téléphoniques serait de plus préjudiciable à une circulation rapide et exacte des informations entre les producteurs et les cellules de crise et structures opérationnelles responsables de la coordination de l'alimentation en eau des populations.

f) D'autres problèmes de fonctionnement

S'ajouteraient aux facteurs précédemment cités une impossibilité de rejet des boues de traitement, qui seraient alors produites en quantités supérieures à la normale, dans une rivière en crue ou dans des réseaux d'assainissement en charge. La fragilisation des stations empêcherait enfin de procéder aux opérations habituelles de maintenance, et interdirait d'intervenir pour remettre en état les parties de leurs équipements qui se trouveraient mises hors service.

\subsection{Vulnérabilité des réseaux de distribution}

A supposer que l'eau puisse être produite en quantité suffisante et en qualité acceptable (ou qu'une information du 
public permette de pallier son absence de potabilité), encore faut-il qu'elle puisse parvenir au consommateur ! Les relais permettant d'acheminer l'eau aux étages de distribution les plus élevés, ou l'échange d'eau entre grands secteurs de distribution, sont eux aussi dépendants de l'alimentation en énergie électrique. Les effondrements des chaussées submergées conduiraient à de multiples casses de canalisations qui ne pourraient être réparées immédiatement, et amèneraient à une surconsommation du réseau alors même que la ressource serait rare.

Au plan qualitatif, s'il n'est pas à craindre de contamination du réseau d'eau potable parisien circulant dans des égouts qui se trouveraient alors en charge, il est à prévoir une intensification du risque des retours d'eau dans le réseau en provenance d'abonnés inondés et mal équipés en dispositifs anti-retour.

\subsection{L'après-crue : de multiples conditions à remplir pour un retour à la normale}

Il est assurément hâtif de penser que le rétablissement des conditions normales de distribution de l'eau potable s'effectuerait sans délai à la fin d'un épisode de crue majeure. Cela supposerait tout d'abord que le fonctionnement de l'ensemble des autres réseaux précédemment énumérés ait été largement rétabli.

Au plan de la qualité de la ressource, les eaux brutes demeurent difficiles à clarifier en période de décrue car elles contiennent encore beaucoup de matières organiques dis- soutes (acides humiques et fulviques notamment), sont plus faiblement minéralisées et généralement froides.

Parallèlement au temps nécessaire à la remise à niveau des stations de production d'eau potable, celui à consacrer à la réparation des fuites des réseaux de distribution ainsi qu'à leur nettoyage et à leur désinfection afin de rétablir la qualité sanitaire de l'eau distribuée se chiffrerait assurément en semaines.

\section{III $\square$ LA GESTION DES CRUES DANS LES SERVICES D'EAU D'ILE DE FRANCE \\ (J.P. Maugendre - Lyonnaise des Eaux)}

Contrairement aux pollutions accidentelles, qui sont brutales et ponctuelles, les crues des rivières de la région parisienne sont à évolution plus lente, ce qui permet d'anticiper la montée des eaux et de prendre des mesures préventives ou conservatoires en temps utile.

\subsection{L'organisation en cas de crue}

L'expérience acquise par les distributeurs d'eau leur a permis d'élaborer des Procédures de Crises ou des Plans d'Alerte qui contribuent, en cas de crues d'une ou plusieurs rivières, à optimiser l'efficacité de leur organisation et à prévoir les moyens exceptionnels, matériels ou humains, à mettre en œuvre. La Compagnie Générale des Eaux, régisseur du SEDIF, tout comme la Lyonnaise des Eaux et la SAGEP, gèrent ces procédures sous Assurance Qualité.
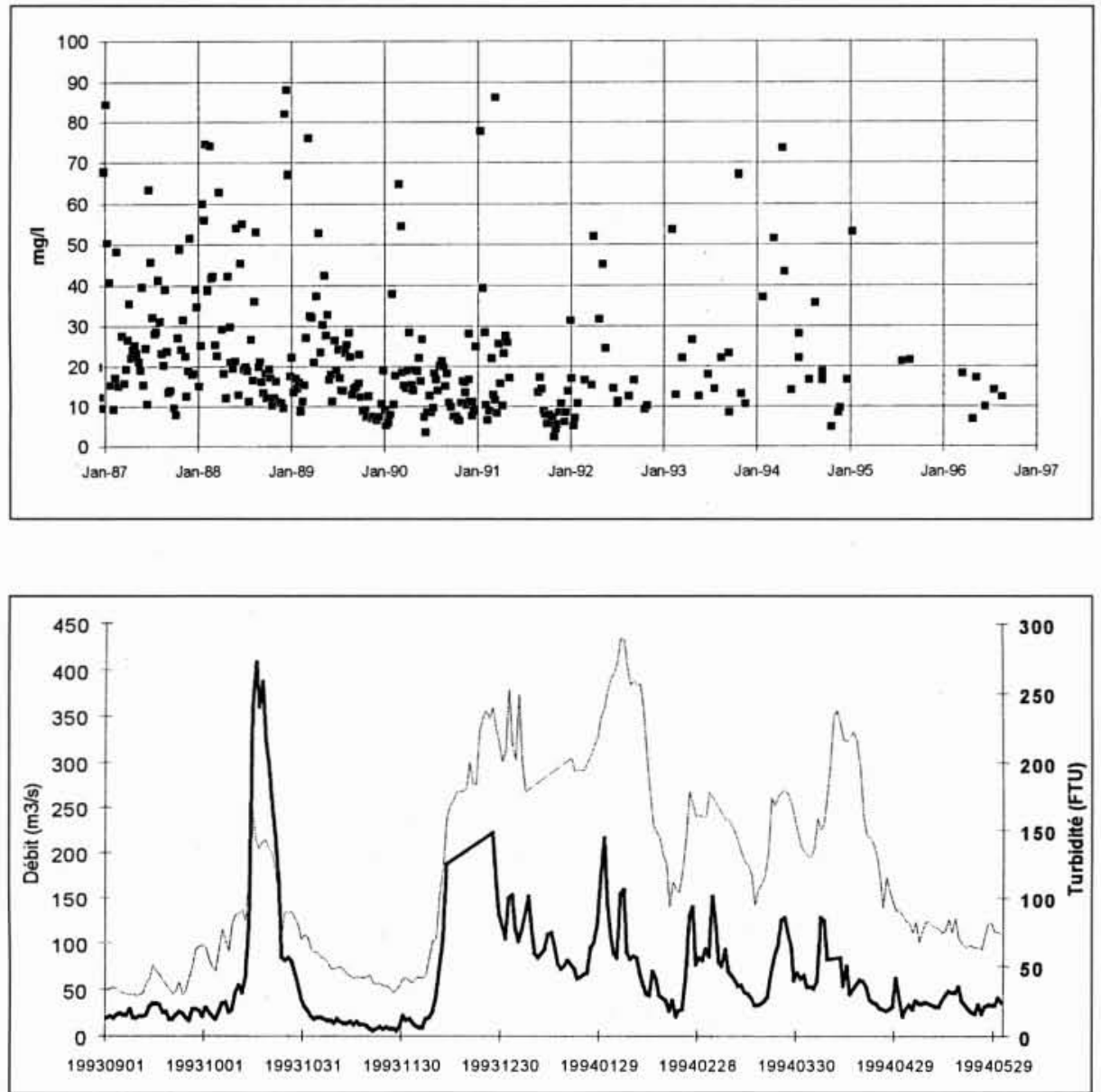

1. Evolution de la teneur moyenne en MES en Seine aval entre 1987 et 1997.

2. Evolution de la turbidité en Marne entre septembre 1993 et mai 1994. 


\begin{tabular}{|l|l|c|c|c|c|}
\hline & & \multicolumn{3}{c|}{ seuils de déclenchement de l'alerte } \\
\hline Rivière & \multicolumn{1}{|c|}{ Lieu } & $\begin{array}{c}\text { débit de la } \\
\text { rivière }\end{array}$ & $\begin{array}{c}\text { niveau de la } \\
\text { rivière }\end{array}$ & turbidité & COT \\
\hline Marne & station d'alerte de Gournay & $300 \mathrm{~m}^{3} / \mathrm{s}$ & - & - & - \\
\hline Marne & Prise d'eau de l'usine de Neuilly/Noisy & - & $35,65 \mathrm{~m}$ & $100 \mathrm{NTU}$ & $4 \mathrm{mg} / \mathrm{l}$ \\
\hline Oise & station d'alerte Ségur & - & - & $100 \mathrm{NTU}$ & $6 \mathrm{mg} / \mathrm{l}$ \\
\hline Oise & Prise d'eau de l'usine de Méry & $300 \mathrm{~m}^{3} / \mathrm{s}$ & $24,45 \mathrm{~m}$ & - & - \\
\hline Seine & Alfortville & $600 \mathrm{~m}^{3} / \mathrm{s}$ & - & - & - \\
\hline Seine & Station d'alerte d'Ablon & - & - & - & $6 \mathrm{mg} / \mathrm{l}$ \\
\hline Seine & Prise d'eau de l'usine de Choisy & - & $32,60 \mathrm{~m}$ & $100 \mathrm{NTU}$ & $5 \mathrm{mg} / \mathrm{l}$ \\
\hline
\end{tabular}

Tableau 4. - Seuils de déclenchement de l'alerte crue dans les usines du Syndicat des Eaux d'lle de France.

Ces plans précisent :

- les critères de déclenchement de la crise,

- les procédures d'information mutuelle entre les différentes unités ou services et avec l'extérieur,

- la liste, graduée suivant la hauteur d'eau dans la rivière, des équipements ou installations qui risquent d'être submergés, des conséquences entraînées par leur arrêt, des mesures à prendre pour pallier ou limiter l'incident.

Ils complètent le Plan Régional d'Alerte en eau potable, actuellement en cours de révision, qui prend en compte, notamment, les risques naturels tels que les crues.

\subsection{L'alerte crue}

Les distributeurs suivent quotidiennement quelques paramètres (niveau de la rivière, turbidité, ...) qui permettent de caractériser une situation de crue.

Dans les usines du SEDIF, les critères de déclenchement du Plan d'Alerte Crue concernent le débit et le niveau de la rivière, la turbidité et le COT de l'eau brute, qui sont mesurés en continu aux prises d'eau ou dans des stations d'alerte situées à quelques kilomètres en amont. A titre d'exemple, le tableau 4 donne les seuils de déclenchement de l'alerte pour les 3 usines du SEDIF.

Les distributeurs d'eau entretiennent des relations privilégiées avec les services de la Navigation, le Service d'Annonce des Crues et l'Institution Interdépartementale des barrages réservoirs du Bassin de la Seine, ce qui leur permet d'être informés régulièrement des prévisions d'évolution à court terme de la crue.

A la Compagnie Générale des Eaux, c'est le Groupe de Protection des Prises d'Eau (GPPE) qui assure cette mission d'information sur le suivi et l'évolution de la crue. En outre, le GPPE entretient des relations constantes avec les responsables des stations d'épuration (ou les industries) situées en amont des prises d'eau afin de prévenir les usines en cas de risque de délestage (ou de pollution).

Les prévisions de crue (débits ou hauteurs d'eau) ne sont actuellement disponibles qu'à très court terme ( 1 jour). Un allongement de ce délai serait très intéressant pour les distributeurs d'eau.

\section{- 3.3 La gestion de la crue au niveau local}

Le rôle des usines est de prendre toutes les dispositions nécessaires pour assurer la continuité de la production d'eau en quantité et en qualité.
Les tableaux des Plans d'alerte, mis à jour régulièrement, indiquent pour chaque niveau atteint par la rivière, l'origine de l'inondation (égouts, chemin de câbles, accès, ...), le matériel touché, les conséquences et les actions à mettre en œuvre.

A titre d'illustration, parmi les actions qui peuvent être mises en œuvre, on peut citer :

- la mise en place de pompes d'épuisement supplémentaires,

- l'obturation de passages de câbles,

- le rehaussement provisoire de coffrets électriques,

- le démontage de certaines motorisations et la mise hors tension de certaines installations,

- la fermeture de certaines vannes d'égouts,

- la construction de murets en parpaings,

- l'obstruction, en maçonnerie, d'ouvertures,

- etc.

La réaction à une crise peut comporter, au-delà des astreintes habituelles, la mobilisation des agents disponibles ou le rappel de certains d'entre eux en congés.

Les usines suivent avec attention l'évolution des stocks de produits chimiques pour la coagulation et des réactifs pour les stations d'alerte. Les taux élevés de matières en suspension et de traitement en réactif floculant entraînent une réduction de l'autonomie en coagulant et une production accrue de boues qui ne peuvent plus toujours être évacuées par les dispositifs habituels. L'accumulation des boues dans les ouvrages, lorsque celles-ci ne peuvent plus être évacuées, est donc également suivie avec attention.

La disponibilité des matériels de secours comme les groupes électrogènes, les motopompes, les pompes d'épuisement, etc., est également surveillée.

Des matériels supplémentaires pourraient aussi être réquisitionnés par la DIREN à notre demande (cette possibilité, testée lors de l'exercice EXINAT, n'a par contre jamais été vérifiée en grandeur réelle).

\subsection{La gestion de la crue au niveau central}

Au niveau central, les organisations des distributeurs d'eau peuvent différer. L'exposé ci-après s'appuie sur l'exemple de la Compagnie Générale des Eaux, régisseur du Syndicat des Eaux d'Ile de France.

La Cellule Qualité Eau définit des objectifs généraux sur la qualité de l'eau traitée (par exemple, turbidité eau décantée $<1$ FTU et COT eau CAG $<2,5 \mathrm{mg} / \mathrm{l}$ ) et aide les secteurs 
à atteindre ces objectifs sans effet secondaire (tel que par exemple : teneur en aluminium trop élevée, indice de saturation négatif, etc...). La Cellule Qualité de l'Eau sollicite, le cas échéant, auprès des Autorités Sanitaires les dérogations nécessaires en cas de dépassement de norme.

Le Centre des Mouvements de l'Eau centralise les informations sur le niveau des trois rivières ainsi que les prévisions actualisées sur minitel (3615 EAUSEINE). Il suit les capacités de production réelle de chaque usine et organise les transferts d'eau entre les secteurs, les échanges d'eau avec les distributeurs voisins et, si besoin, fait reporter les travaux qui diminueraient la capacité de production. Il est également chargé de l'information de la Direction et de l'autorité délégataire, et de l'export de l'alerte vis-à-vis des autorités administratives.

Des interconnexions à grand débit ont été construites entre les usines de production. Une liaison Oise-Marne supplémentaire viendra compléter la liaison existante et les 2 liaisons Seine-Marne déjà opérationnelles depuis plusieurs années.

Ces interconnexions ou intercommunications autorisent en cas de nécessité le ralentissement d'une usine de production. L'onde de crue maximale n'est généralement pas simultanée au droit des différentes usines de production (situées plus à l'amont ou l'aval d'une même rivière, ou sur des rivières différentes). Une bonne gestion des disponibilités des usines et des interconnexions permet donc de satisfaire généralement les besoins et d'optimiser la qualité de l'eau produite.

\section{- 3.5 Le retour à la normale}

L'arrivée de la décrue constitue un soulagement pour les hommes qui ont été fortement sollicités, mais ils ne peuvent malheureusement pas être démobilisés immédiatement, même en cas de crue moyenne :

- la qualité de l'eau ne s'améliore pas instantanément,

- les stocks de coagulants sont à reconstituer,

- les surplus de boues non évacués sont à éliminer,

- des sous-sols inondés sont à pomper et à nettoyer,

- il faut préparer le debriefing de la crise, afin d'en retenir les enseignements et de mettre à jour les plans d'alerte, - etc.

Tout cela demandera 8 à 10 jours d'efforts supplémentaires.

En cas de crue plus sévère, si des équipements, électriques notamment, ont été submergés, leur remise en état de fonctionnement peut nécessiter un délai d'une à plusieurs semaines :

- remplacement des roulements des pompes qui ont été inondées,

- dépose, nettoyage et étuvage des moteurs ou armoires électriques qui ont été inondés,

- remplacement et/ou reprogrammation des automates programmables.

\subsection{Entre deux crues}

Comme pour toute crise, il convient de s'y préparer avant d'y être confronté.

Les procédures sont donc régulièrement testées et améliorées pour en évaluer la pertinence et les tenir à jour, soit lors de l'analyse de crues réelles comme en 1994 et 1995, soit lors d'exercices de simulation, internes ou externes (par exemple exercice LUTECE en 95 : scénario crue de 1910, préparé mais annulé, ou exercice EXINAT en 1996 : scénario crue de 1955 mêlée à d'autres événements).

Les expériences vécues ou les exercices ont permis aussi de répertorier divers travaux sur les installations qui permettraient de retarder les dysfonctionnements en cas de montée des eaux.

Ainsi, la Lyonnaise des Eaux a établi pour chaque site d'installations un bilan précis et chiffré des travaux de modifications à réaliser pour garantir le fonctionnement des installations pour plusieurs niveaux de crues historiques et qui seront progressivement programmés.

De la même façon, la Compagnie Générale des Eaux, à la suite de la crue de l'Oise de 1995, a proposé au Syndicat des Eaux d'Ile de France, pour le projet d'extension de l'usine de Méry-sur-Oise :

- de placer le radier de l'ensemble des bâtiments à la cote $26,00 \mathrm{~m}$, soit sensiblement au dessus du niveau de la crue centennale $(25,79 \mathrm{~m}$ en 1926$)$,

- de surélever le poste de transformation électrique à la cote $27,00 \mathrm{~m}$ afin que le fond de tous les caniveaux électriques ne descendent pas en dessous de $26,00 \mathrm{~m}$,

- de concevoir les points singuliers et notamment les pénétrations de câbles dans les différents bâtiments afin qu'ils ne puissent être à l'origine d'infiltrations.

\section{IV - CONCLUSION}

La vulnérabilité par rapport aux crues des installations de production d'eau potable d'Ile de France fait apparaitre un bilan besoin-ressources plutôt favorable au plan quantitatif.

Les politiques préventives menées et les organisations de crise mises en place par les collectivités locales responsables et les distributeurs d'eau ont fait que la distribution d'eau en Ile de France n'a pas été perturbée lors des crues majeures des dernières décennies.

Toutefois, lors de crues très importantes, les installations et leur conduite deviennent plus vulnérables à divers incidents, et la dégradation de la qualité de l'eau brute des rivières reste une forte préoccupation, même lors de crues relativement modestes.

En cas de crue de type 1910 , tout porte à croire que la multiplicité des dysfonctionnements du système économique dont dépend la production et la distribution d'eau potable nécessiterait une coopération étroite entre les cellules de crises des distributeurs d'eau et celles des pouvoirs publics, dans le cadre d'une gestion globale de crise. 